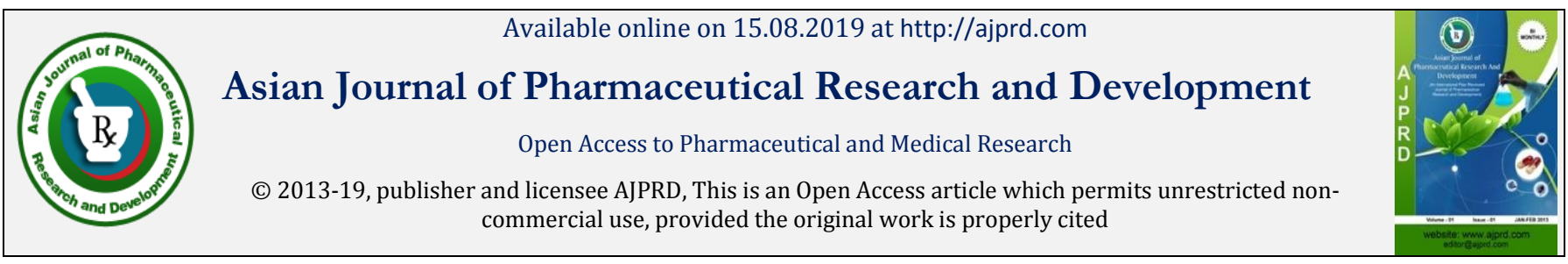

Open $\mathcal{O}_{\text {Access }}$

Review Article

\title{
AN OVERVIEW ON POLYCYSTIC OVARIAN SYNDROME
}

\section{Yedulapuram S H ${ }^{1}$, Gunda $\mathbf{M}^{2}$, Moola $\mathbf{N} \mathbf{R}^{3}$ and Kadarla $\mathbf{R} \mathbf{K}^{*}$}

Department of Pharmacy Practice, Sree Chaitanya institute of Pharmaceutical Sciences Thimmapur, Karimnagar, Telangana, India-505527

\begin{abstract}
A B S T R A C T
World Health Organization (WHO) estimates that PCOS has affected 116 million women (3.4\%) worldwide in 2012 . Globally, prevalence estimates of PCOS are highly variable, ranging from $2.2 \%$ to as high as $26 \%$. In India, experts claim $10 \%$ of the women to be affected by PCOS and yet no proper published statistical data on the prevalence of PCOS in India is available. Polycystic Ovary Syndrome (PCOS) is an endocrine metabolic disorder characterized by multiple hormonal imbalances representing diverse clinical presentations dominated by clinical and biochemical signs of hyperandrogenism which results in short and long term consequences in female health. A defect of the ovarian cells (most likely theca cells) is the underlying cause of PCOS, resulting in excessive androgen synthesis and the clinical and biochemical symptoms of the disease. In the literature, reference is made to the participation of genetic factors, including ethnicity; there is a higher frequency of PCOS in Spanish, Native American and Mexican women. The patient seeks a dermatology consultation for one or more complaints like acne, hirsutism, alopecia, acanthosis nigricans, skin tags and occasionally, darkening of complexion with weight gain. . If irregular menstrual cycles or primary infertility are the main complaints, the patient may consult a gynaecologist. Polycystic ovaries found on ultrasound scanning will often have no clinical effects, but PCOS is the most common diagnosis made in women presenting with amenorrhoea, oligomenorrhoea or heavy, irregular and prolonged periods. It is the commonest cause of hirsutism and of infertility due to anovulation. Women with PCOS have increased concentrations of circulating androgens and there is a marked association with insulin resistance, dyslipidaemia, obesity, gestational diabetes, type 2 diabetes and heart disease
\end{abstract}

KEYWORDS: Polycystic Ovary Syndrome, hyperandrogenism, metabolic disorder.

A R T I C L E I N F O: Received 2 May 2019; $\quad$ Review Completed 27 July 2019; Accepted 31 July 2019; $\quad$ Available online 15 August 2019

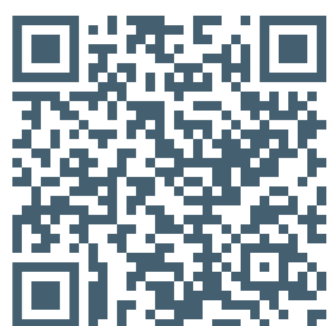

Cite this article as:

Yedulapuram S H, Gunda M, Moola N Rand Kadarla R K, An Overview on Polycystic Ovarian Syndrome, Asian Journal of Pharmaceutical Research and Development. 2019; 7(4):72-80, DOI: http://dx.doi.org/10.22270/ajprd.v7i4.514

*Address for Correspondence:

Dr. Kadarla Rohith Kumar, Asst Professor, Department of Pharmacy Practice,Sree Chaitanya institute of Pharmaceutical Sciences,Thimmapur, Karimnagar,Telangana, India

\section{INTRODUCTION}

$\mathrm{P}$ olycystic Ovary Syndrome (PCOS) is an endocrine metabolic disorder characterized by multiple hormonal imbalances representing diverse clinical presentations dominated by clinical and biochemical signs of hyperandrogenism which results in short and long term consequences in female health. It has a tremendous negative impact on the women's physiology and metabolism leading to metabolic alterations i.e., insulin resistance, hyperinsulinemia, abdominal obesity, hypertension and dyslipidaemia culminating as serious long term consequences such as T2DM, endometrial hyperplasia and CVS diseases ${ }^{1}$ The prevalence rate of PCOS in India was found to be $30 \%$. Teenagers are being most affected. PCOS is described as an oligogenic disorder with an interaction of genetic and environmental factors which determine the heterogenous clinical and biochemical phenotype expression in PCOS women. ${ }^{2}$ The expression of PCOS is mainly due to polymorphism of genes like FBN-3, LHR, TNF- alpha and IL's. FBN-3 is involved in the regulation of 
TGF signaling. LHR have a role of LH, T stimulation, follicle development, LH surge induced ovulation in ovaries and LH stimulation of adipogenesis in adipocyte. ${ }^{3}$ Diet, lifestyle and physical activity also have an influence on women health. LH/FSH ratio is elevated in PCOS women. The three common factors associated with PCOS are anovulation, clinical and biochemical hyperandrogenism (hirsutism, acne, alopecia) and polycystic ovaries. Insulin resistance, obesity, CVD, cancer, infertility, miscarriage, preeclampsia and gestational DM are commonly developed complications in women with PCOS. Treatment is mainly based on clinical presentation of the patients i.e., menstrual irregularities, hyperandrogenism and anovulation.

Polycystic ovaries found on ultrasound scanning will often have no clinical effects, but PCOS is the most common diagnosis made in women presenting with amenorrhoea, oligomenorrhoea or heavy, irregular and prolonged periods. It is the commonest cause of hirsutism and of infertility due to anovulation. Women with PCOS have increased concentrations of circulating androgens and there is a marked association with insulin resistance, dyslipidaemia, obesity, gestational diabetes, type 2 diabetes and heart disease. In addition, it is an established cause of endometrial hyperplasia and it is therefore linked to endometrial cancer. The short- and long-term consequences of PCOS represent an increasing burden on health resources. In recent years there has been increasing consensus about the criteria required to establish the diagnosis of PCOS. Two out of the three features below are used when other causes of those clinical features have been excluded ${ }^{4}$ Exclusion of these other, much rarer, causes often needs no more than a routine clinical assessment. The three features are:

\section{Anovulation or oligo-ovulation;}

The presence of polycystic ovaries on pelvic ultrasonography;

Clinical and/or biochemical signs of hyperandrogenism. While awareness of PCOS, its diagnosis and associated morbidity is high, its basic pathophysiology is often poorly understood. This article presents a framework for teaching and increasing understanding about the causes and management of PCOS ${ }^{36}$

\section{EPIDEMIOLOGY:}

The prevalence of PCOS in India was found to be $30 \% .{ }^{4}$ In middle east countries( Iran and Turkish), China, North California, Australia, Africa the prevalence of PCOS was found to be $6.1 \%, 5.6 \%, 0.83 \%, 8.7 \%$ and $7.4 \%$ respectively. ${ }^{5}$ Teenagers are being most affected $(50 \%)$. Almost 75\% women with PCOS develop insulin resistance and $10 \%$ of them develop diabetes by age 40 . Risk of developing DM in PCOS patients is $15.4 \%$. ${ }^{5}$ The prevalence of PCOS in women with dysmenorrhea was $40 \%$. The prevalence of PCOS was lower in black women compared to white. According to body weight, the prevalence of PCOS is under weight $(8.2 \%)$, Normal (9.8 $\%)$, Over weight $(9.9 \%)$, Obese $(9 \%){ }^{4}$

\section{CAUSES}

A defect of the ovarian cells (most likely theca cells) is the underlying cause of PCOS, resulting in excessive androgen synthesis and the clinical and biochemical symptoms of the disease. ${ }^{6,7}$ In the literature, reference is made to the participation of genetic factors, including ethnicity; there is a higher frequency of PCOS in Spanish, native American and Mexican women. In their original description of the syndrome, Stein and Leventhal emphasized that a high ratio of luteinizing hormone (LH) to follicle-stimulating hormone (FSH) is one of the basic disorders. It has also been suggested that the underlying causes of PCOS include increased frequency of gonadotropin-releasing hormone $(\mathrm{GnRH})$ pulses that stimulate the theca cells to produce androgen; decreased levels of FSH (and thus a defect in the late luteal and early follicular phases); insulin resistance via a post-receptor defect in the fat tissue and skeletal muscles (abnormal phosphorylation of tyrosine kinase); pancreatic beta-cell dysfunction; and obesity. ${ }^{6,8}$ It is often impossible to determine definitively what is a cause and what is an effect in the development of PCOS. In addition, it is generally recognized that obesity increases menstrual disorders and hyperandrogenism, while weight reduction reduces the clinical signs. Reduced insulin sensitivity is an important issue in both obese and underweight women with PCOS; it is estimated that $50-70 \%$ of women with the condition show insulin resistance of varying intensities. ${ }^{8}$ Genetic factors The influence of genetic factors was highlighted by Davies et al., who proved that mothers of women with PCOS are more likely to have a cardiovascular disease and that their risk of hypertension is twice as high as mothers of women without PCOS, while fathers of women with PCOS are twice as likely to have heart disease and 4 times more likely to have experienced cerebral stroke. ${ }^{10}$ Tan et al. emphasized the increased likelihood of insulin resistance (IR) associated with certain genes (such as INSIG2 and MC4R) and the particular impact of TCF7L2 SNP on the development of diabetes mellitus type 2 (DM2) and body weight gain in patients with PCOS (a per-allele weight gain of $1.56 \mathrm{~kg} / \mathrm{m} 2){ }^{6}{ }^{6}$ The etiology of IR was also discussed by Fica et al., who, while highlighting the complex mechanisms of PCOS, identified insulin receptor autophosphorylation, reduced levels of phosphatidylinositol-3-kinase in muscle tissue and visceral adiposity as probable mechanisms. ${ }^{7}$

\section{Hyperinsulinemia/insulin resistance}

Hyperinsulinemia in combination with pancreatic beta cell dysfunction results in an increased risk of many diseases, including type 2 diabetes, hypertension, dyslipidemia, endothelial dysfunction, atherosclerosis and cardiovascular diseases. Insulin also stimulates the theca cells of the ovary to produce excessive testosterone, which is responsible for the clinical symptoms of hyperandrogenism (acne, hirsutism, alopecia). ${ }^{8}$ Cardiovascular risk is also elevated in women who are chronic smokers, as demonstrated in a recent work by Marotti et al. ${ }^{9}$

\section{Inflammation}

The role of inflammation in PCOS has been the subject of a number of studies, and direct correlations have been found between increased levels of inflammation markers (CRP, 
ferritin, leukocyte TNF- $\alpha$, IL-6, IL-18) and the development of PCOS. Other contributors include elevated levels of PAI1 and free fatty acids, influencing excessive phosphorylation of serine residues, leading to a rise in insulin resistance. ${ }^{9}$ Newly emerging issues include a pathogenic correlation of the markers of iron overload with PCOS. Increased levels of ferritin and transferrin and a higher frequency of the HP2/HP2 genotype of the haptoglobin $\alpha$ chain have been observed, causing a reduction of anti-inflammatory cytokines and antioxidant molecules, leading to a state of chronic inflammatory response ${ }^{16,17}$

\section{CLINICAL FEATURES:}

The patient seeks a dermatology consultation for one or more complaints like acne, hirsutism, alopecia, acanthosis nigricans, skin tags and occasionally, darkening of complexion with weight gain. If irregular menstrual cycles or primary infertility are the main complaints, the patient may consult a gynaecologist. An endocrinologist may be consulted for hirsutism and the metabolic syndrome. Very rarely do patients present with all the clinical signs and symptoms of PCOS and some may not be forthcoming with information of concurrent treatment from a gynaecologist or an endocrinologist. An alert clinician should be able to link the symptoms together, pointing to a possible underlying defect of hyperandrogenism.

\section{Acnevulgaris}

Patients with PCOS complain of inflammatory acne minimally responsive to conventional line of treatment. Even if responsive, lesions promptly recur on stopping treatment, necessitating treatment with oral isotretinoin and/or hormonal therapy. An important feature seen in these patients is the development of multiple closed comedones which rapidly transform into tender, lumpy nodules, distributed in the lower half of face and jaw-line (V distribution)fig- 1 . These tend to persist beyond the usual course of 5-7 days. A pre-menstrual flare is also common. Acne lesions may not only be localized to the face, but may also be present on the chest, shoulders and back. Prompt relapse after stopping the treatment, strongly suggests a hormonal basis. Patients may in addition, have a history of irregular periods, and evidence of hirsutism, alopecia, or a positive family history of PCOS. The severity of hirsutism may not be matching the severity of acne, and would be dependent on the balance of activity between the alfahydroxy type 2 vis-a-vis type $1 .{ }^{18}$

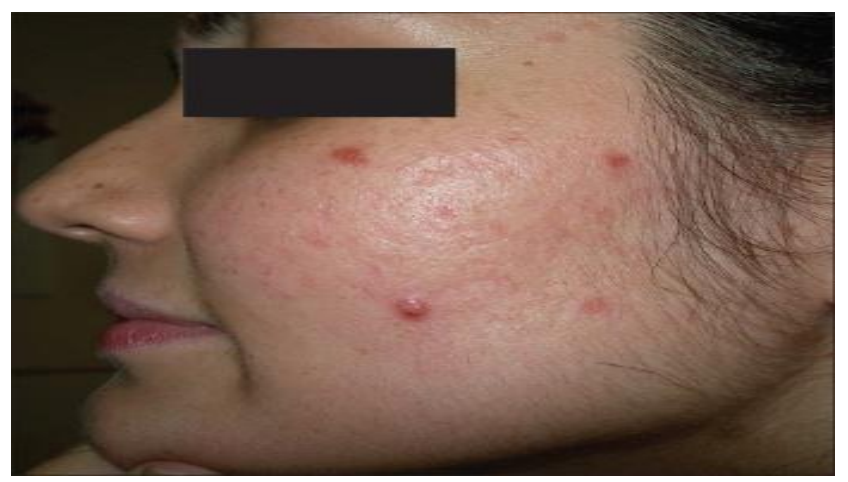

Figure 1: Acne with hirsutism in a patient with PCOS

\section{Hirsutism}

Excessive facial hair is a racial trait for the Indian subcontinent, running within families and especially strong in certain ethnic groups. This should be kept in mind while evaluating patients complaining of excessive facial/body hair. Androgens affect various aspects of follicular activity. Acting via androgen receptors and secretory factors, they increase the growth rate, diameter and melanization of hair in androgen-sensitive areas. It is these thick, coarse, terminal hair, in androgen-dependent areas which are unsightly on a female ${ }^{19}$ and point to an underlying hyperandrogenic state Fig: 2. Evaluation of the degree of hirsutism is done by adopting a modified Ferriman-Galway score which evaluates 9 body areas on a scale of 1 to 4 . The total scores are significant if more than 6 to $8^{18,20}$

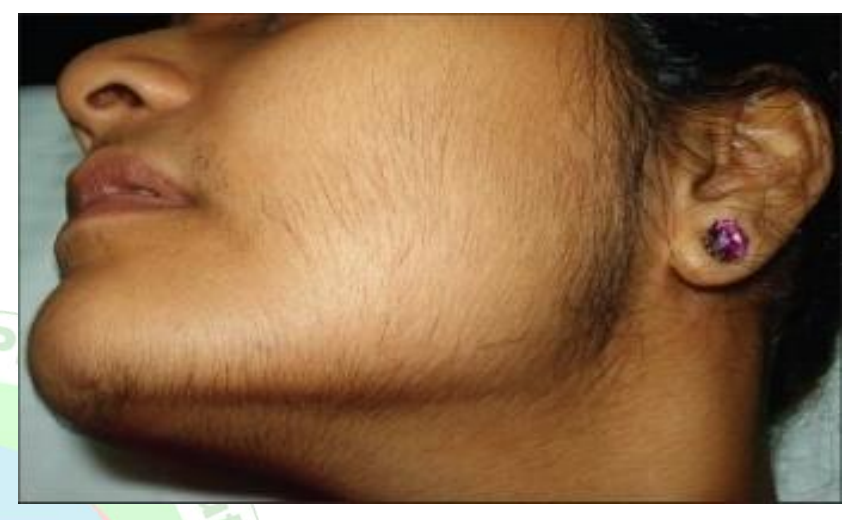

Figure 2: Hirsutism in a patient with PCOS

\section{Alopecia}

Not all cases of female pattern hair loss (FPHL) may be of androgenic origin. ${ }^{[21]}$ Patterned hair loss in PCOS may be difficult to distinguish from those secondary to other hyperandrogenic states Fig:3. Various clinical presentations include those described by Ludwig (diffuse), Hamilton (male pattern), and Olsen (frontal accentuation ${ }^{21}$ Women with early onset FPHL are much more likely to have an associated hyperandrogenism. Hormonal influences convert terminal to vellus hair, making the scalp appear bald.

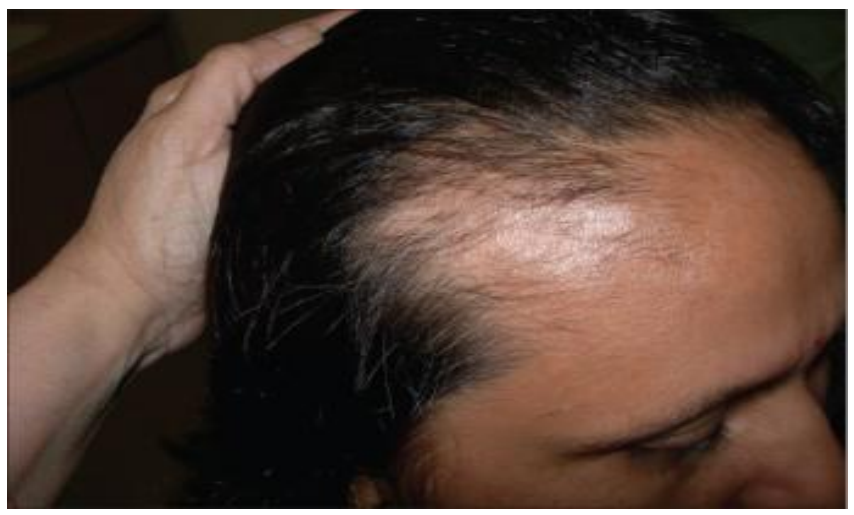

Figure 3: Patterned hair loss with bi-temporal recession in a patient with PCOS

\section{Acanthosis nigricans}

Typically thick dark velvety skin situated on the nape of the neck, axillae, groins and other frictional areas may often be the first clue of insulin resistance Fig 4. The thickening occurs due to the stimulation of tyrosine kinase growth 
factor - signaling pathways in the epidermis. Insulin - like growth factor receptor 1 (IGF1R) is present in many tissues including the epidermis and ovary. High levels of insulin directly or indirectly stimulate the IGF1R resulting in the skin changes. Skin tags in the frictional areas like the neck, axillae, groins, infra mammary or even under a pendulous abdominal fold are common, especially in obese individuals.

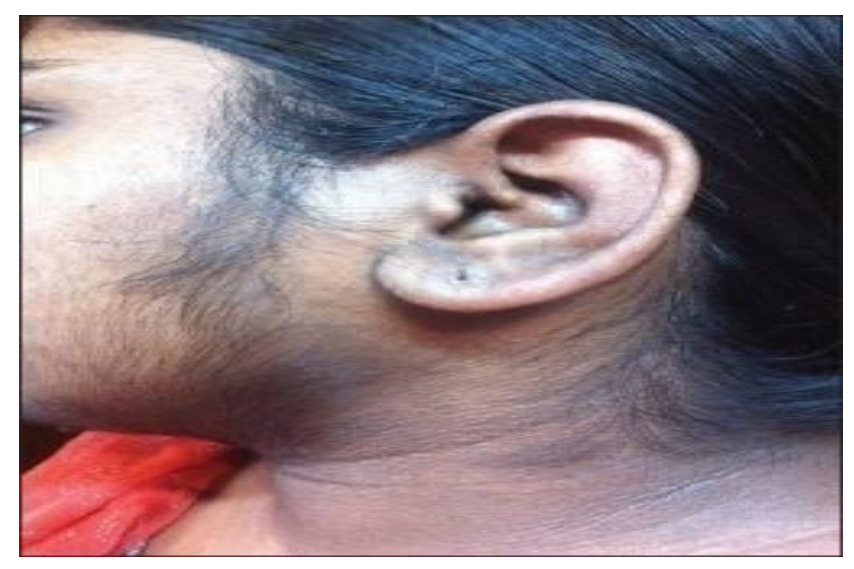

Figure 4: Acanthosis nigricans and hirsutism in an obese girl with PCOS

\section{Irregular menses and infertility}

PCOS is characterized by chronic menstrual irregularities or changes in menstrual pattern with reduced fertility. The anovulatory or oligoovulatory cycles result in continuous endometrial stimulation with estrogens, resulting in endometrial hyperplasia, thus increasing the risk of endometrial cancers ${ }^{22}$

\section{PATHOPHYSIOLOGY:}

PCOS is a complex disorder and there is no definite explanation. PCOS is thought to be caused by hormonal imbalance. Women with PCOS show about twice the levels of androgens compared to normal healthy women. There are many research theories for PCOS. Some suggest it as a genetically inherited disease, some express that the ovaries produce too much androgens due to defect in the hypothalamus, some theorize ovarian abnormality, another suggests culprit is hyperinsulinemia which lead to hyperandrogenism. In PCOS, many physiological events within the ovarian cycle and folliculogenesis are dysregulated. The very beginning of folliculogenesis is compromised due to high levels of anti mullerian hormone $(\mathrm{AMH})^{1}$ As there are high levels of AMH in PCOS women, ovarian physiology is disrupted and also results in worse fertility outcome. ${ }^{1}$ Theca cells secrete high levels of androgens due to an intrinsic activation of steroidogenesis in the absence of trophic factors. This intirinsic defect in the theca cells also has an effect on granulosa cells which produce four times more AMH levels. Studies also revealed an elevated number of follicles, primarily pre antral and small antral follicles. Together with an defect apoptotic processes in maturing follicle will increase the follicle count in PCOS women. ${ }^{23}$ Another typical feature of PCOS are feedback disturbances in hypothalamus- hypophysis-ovary axis (HHOA) increased frequency and amplitude of GnRh and LH pulsatile secretion. ${ }^{24}$ Higher levels of this hormones induce ovarian theca cells to secrete androgen excess. Hyperandrogenemia inturn causes decrease in feedback sensitivity to both estradiol and progesterone in gonadotropic-hypothalamic cells, reinforcing GnRh and $\mathrm{LH}$ hypersecretion ${ }^{24}$ Hyperandrogenemia plays a pivotal role in the development and progression of PCOS. The pathophysiological aspects of PCOS are clearly explained by following- endocrine dysfunction, reproductive dysfunction, metabolic dysfunction and biochemical dysfunction.

\section{Reproductive dysfunction:}

Women with PCOS frequently present with reproductive dysfunction. The exact pathogenesis of anovulation is not clearly known but many mechanisms like LH hypersecretion, hyperandrogenemia, hyperinsulinemia, obesity, decreased plasminogen activator inhibitor (PAI) activity and endothelial dysfunction are interlinked. Menstrual irregularity is the most common gynecological presentation of PCOS. 85-90\% of women with PCOS are oligomenorroheic and 30-40\% women are amenorrheic ${ }^{1}$ Polycystic ovaries display more number of follicles compared to normal ovaries suggesting follicular development disturbances. Perturbations in gonadotropin secretion in PCOS such as decreased FSH and increased LH results in abnormal follicular dynamics manifesting in anovulatory infertility. Decreased FSH levels results in follicular growth arrest at 2-8 $\mathrm{mm}$ stage and produce estrogen and inhibin by multiple, small follicles which inturn inhibit FSH secretory dynamics preventing selection of dominant follicle and thus contributes to impaired follicular development ${ }^{1}$

\section{Endocrine Dysfunction:}

PCOS is marked by abnormalities of both feed forward and feedback signaling between $\mathrm{GnRH} / \mathrm{LH}$ and ovarian androgens. The key endocrine abnormalities of reproductive axis include accelerated GnRh pulsatile activity, LH hypersecretion, theca-stromal cell hyperactivity and hypofunction of the FSH- granulosa cell axis ${ }^{1}$ 


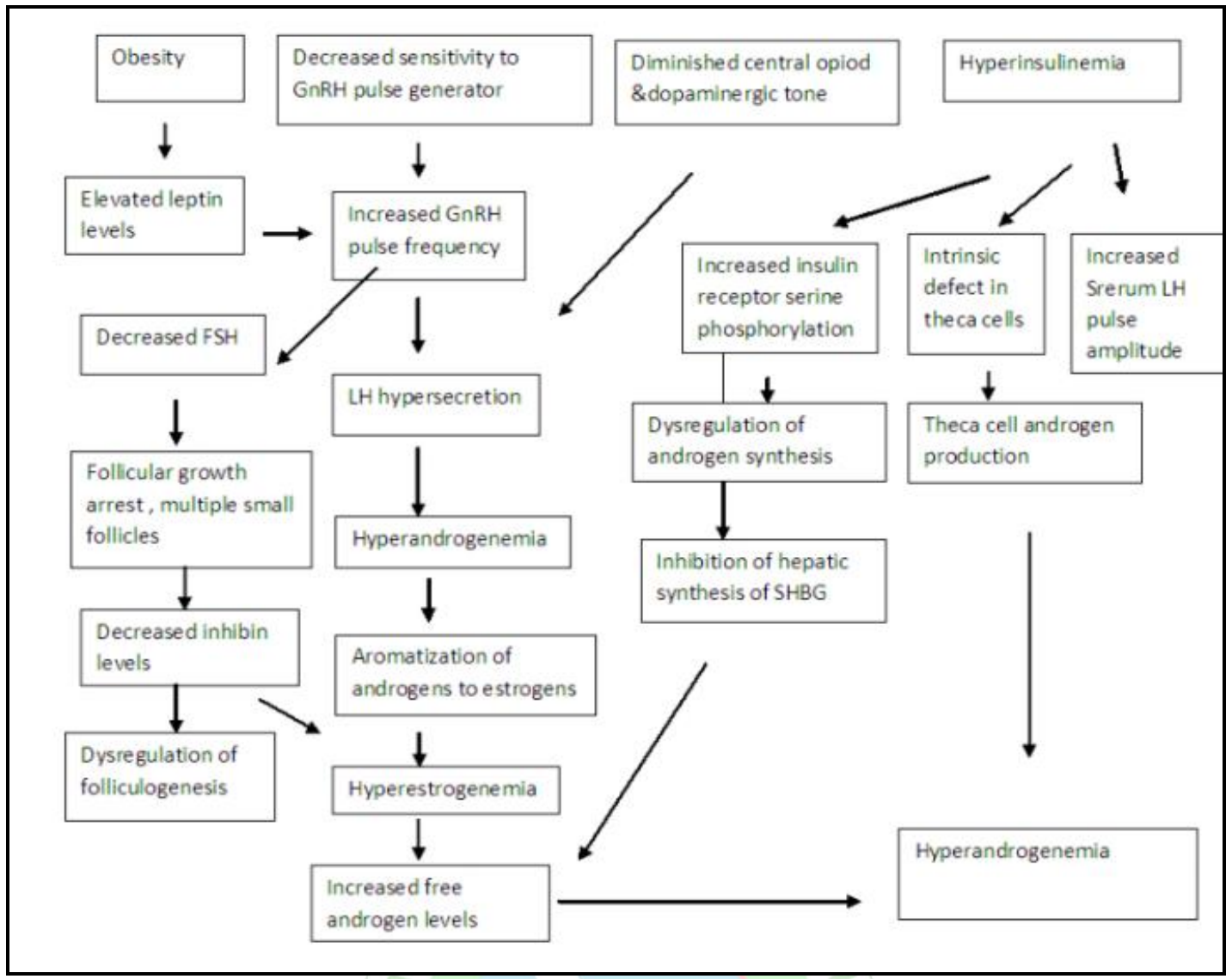

Figure: 5 Endocrine dysfunction in pcos ${ }^{1}$

\section{COMPLICATIONS:}

Insulin resistance Insulin resistance develops most commonly in PCOS women which may lead to dyslipidemia. IR increases VLDL secretion thereby decreasing its elimination and also chylomicrons elimination which may further leads to production of triglycerides. IR also leads to clearance of HDL-C constituent which is apolipoprotein-a, and leads to decreased levels of HDL-C. Postbinding decrease in the phosphorylation of tyrosine residues and increased serine phosphorylation of the insulin receptor cause resistance to metabolic actions of insulin. Increase in serine phosphorylation decreases the response of insulin receptor to its substrate and increases the $\mathrm{P} 450 \mathrm{C} 17$ activity, which is an important enzyme involved in the adrenal and ovarian steroidal synthesis ${ }^{25}$.

Obesity: Obesity is the important feature of the PCOS. PCOS women have excess of androgens which may lead to high visceral and subcutaneous fat. Androgens involves in the dysregulation of appetite. There is decreased production of gastrointestinal satiety peptide cholecystokinin and also dysregulation in secretion of ghrelin, which is an appetite regulating hormone in PCOS women. Their levels are increased in PCOS women, so obesity is more frequently $\operatorname{seen}^{25,26 .}$

Cardiovascular diseases Patients with PCOS have high levels of biomarkers such as $\mathrm{C}$ - reactive protein and Lipoprotein A. ${ }^{23}$ the risk of CVD in PCOS is hyperinsulinemia. One mechanism of insulin resistance is direct atherogenic action which results in early onset of cardiovascular dysfunction. The other risk factors for CVD in PCOS is impaired glucose tolerance and $\mathrm{DM}^{25,27}$

PCOS women have elevated concentration of plasminogen activator inhibitor or fibrinogen that results in impaired fibrinolysis.

Cancer Women with PCOS has high risk of developing endometrial cancer. Prolonged anovulation triggers unopposed estrogen secretion which increases risk of endometrial carcinoma. Other risk factors associated with endometrial cancer includes nulliparity, infertility, obesity and DM. More than 3 months of interval between menstruation is associated with endometrial hyperplasia. Studies link the use of drugs such as clomiphene citrate increases the risk of developing ovarian cancer, multiple ovulation, early menarche, late menopause and nulliparous increases the risk of ovarian cancer ${ }^{23,27}$

Infertility: Infertility is 10 times more common in PCOS women compared to healthy controls. As PCOS is the common cause of ovulatory disorder the risk of infertility increases due to associated endocrine and gynaecological abnormalities that affect the ovarian function ${ }^{23}$ Endometrial abnormalities affect the implantation in PCOS women ${ }^{28}$

Gestational complications: - The risk of gestational complications such as miscarriage, gestational DM and preeclampsia increases in women with PCOS. Several factors such as infertility treatment, IR, multiple pregnancies, obesity, metabolic dysfunction, inflammation 
and placental alterations results in increased incidence of gestational complications in PCOS women. The risk of developing miscarriage, preeclampsia, gestational DM is $30-50 \%, 10-15 \%$ and $20-30 \%$ respectively ${ }^{23,29}$

Psychological disorders Women with PCOS have more chance of developing psychological disorders. They may have anxiety, depression, binge-eating, disorder, bipolar disorder, psychosexual dysfunction. Depression is more commonly seen in teenage girls because of difficulty in managing the overweight. Because of the infertility, miscarriage, sub fertility problems adult women also disturb psychologically. Psychiatric disorders also develop due to insufficient sleep which is seen in the PCOS women ${ }^{23}$

RISK FACTORS: According to one study assessment of risk factors among subjects in PCOS and non-PCOS groups The number of study participants who had a positive family history of PCOS in PCOS group and non-PCOS group are $42(60.8 \%)$ and $106(58.5 \%)$ respectively. It was observed that the participants with family history of PCOS carry a little higher risk [RR 1.07 (CI 0.709-1.619)] of development of PCOS compared to participants without a family history. Majority of the participants in PCOS [60 (86.9\%)] and nonPCOS [170 (93.9\%)] groups were consuming fast food diet less than or equal to 3 days in a week and only 9 and 11 participants were consuming fast food diet more than 3 days in a week in each PCOS and non-PCOS group. It was observed in our study that participants consuming fast food diet for more than 3 days/ week have 1.7 times more risk of developing PCOS compared to those consume fast food diet for less than 3 days/week also this risk is statistically significant $(\mathrm{p}=0.044)$. Majority of study participants in both the groups were involved in physical exercise as less as one day in a week to maximum of all the seven days of the week. It was observed that there was no statistically significant difference $(\mathrm{p}=0.998)$ exists between the individuals who are engaged in physical exercise less than 3 days in week with that of individuals engaged in physical exercise for more than 3 days in a week. The risk was found to be almost equal between the two groups for development of PCOS. The BMI of study participants both in PCOS and non-PCOS groups was calculated and further categorized into normal (BMI $18.6-24.9 \mathrm{~kg} / \mathrm{m} 2$ ), overweight (BMI 25$29.9 \mathrm{~kg} / \mathrm{m} 2$ ) and obese (BMI $30-40 \mathrm{~kg} / \mathrm{m} 2$ ) following World Health Organization criteria. There was no statistically significant difference between individuals with normal BMI and overweight; however a statistically significant difference $(\mathrm{p}=0.030)$ was observed between individuals with normal BMI and obese, with 1.74 times more risk of PCOS among obese participants. Participants in both the groups were categorized as individuals with waist circumference $\leq 80$ centimeters and with $>85$ centimeters. It was observed that there was no statistically significant difference was found between these two groups with respect to development of PCOS. 34

\section{TREATMENT:}

Non pharmacological management Non pharmacological treatments have a great impact on the PCOS patient. Effective approaches to nutrition and exercise improves endocrine and reproductive function and minimizes CVS risk associated with PCOS. Moderate weight losses (greater than 5\%) have shown beneficial effects on well being, insulin sensitivity and CVS risk presentation by improving glucose tolerance, reproductive function. Weight loss is the first line treatment for obese patients. Weight loss occurs when the energy intake is less than energy expenditure. Stress reduction is other major lifestyle modifications which improves the physical and mental status of an individual.

\section{Diet}

Fiber rich foods such as broccoli, brown rice, berries, lentils. Fiber rich foods combat insulin resistance by slowing digestion and reducing glucose impact on the blood. Consume lean protein sources like tofu, fish, chicken and anti inflammatory foods like cabbage, kale, almonds, olive oil, tomatoes, fish high in omega 3 fatty acids (salmon, sardines). Foods that should be avoided include foods high in refined carbohydrates like muffins and white bread, sugary snacks, drinks, processed foods and red meat. Pasta, noodles should be restricted as they are high in carbohydrate and low in fibres. Alcohol intake, red meat, foods containing hydrogenated oils, gluten grains, white flour, white sugar, caffeine may aggrevate the symptoms. Recommended calorie intake Consumption of unsaturated fatty acids have shown to improve insulin sensitivity. Long chain polyunsaturated fatty acids (PUFA 'S) also have beneficial effects on endocrine and metabolic parameters. Follow mediterrenean diet. Foods with high glycemic index delivers carbohydrates rapidly resultin in glycemic overload associated with DM. consumption of foods containing low glycemic index is beneficial. Individual should encourage intake of macronutrients includes $40 \%$ carbohydrates, $30 \%$ proteins, $30 \%$ or less of fats with exercise. Studies confirmed less frequent huge or large eating patterns are associated with fat buildup hence frequent small meals is preferable or desirable. Avoid trans fat as they have been linked to anovulation and infertility outcomes in PCOS women. Low calorie rich diet with energy restriction of $500600 \mathrm{kcal} / \mathrm{day}$ with reduced carbohydrates intake are recommended to obese women with PCOS. According to American heart association guidelines, dietary fiber intake should be 25-30 g/day, MUFA diet should be $24 \mathrm{~g} /$ day $^{30,31}$

Lifestyle changes Diet and calorie restriction works better when suitable exercise programme is maintained. 60-75 minutes of moderate to high intensity physical activity promotes weight loss and show positive impact on the disease. Performing 30 minutes aerobic sessions/week for 12 weeks is the standard exercise pattern. Prolonged or vigorous exercise may enhance weight loss. Types of exercises include aerobic exercise, endurance exercise, resistance training, exercises to increase flexibility PCOS women are advised to perform 90 minutes of aerobic exercise/week at moderate intensity of $60-70 \%$ to improve reproductive and cardiometabollic outcomes. Behavioural counseling and meditation session are advantageous for stress management and weight loss. Behavioural counseling includes regular personnel or group sessions of motivational support $^{32,33}$ 
Table 1: Pharmacological management:

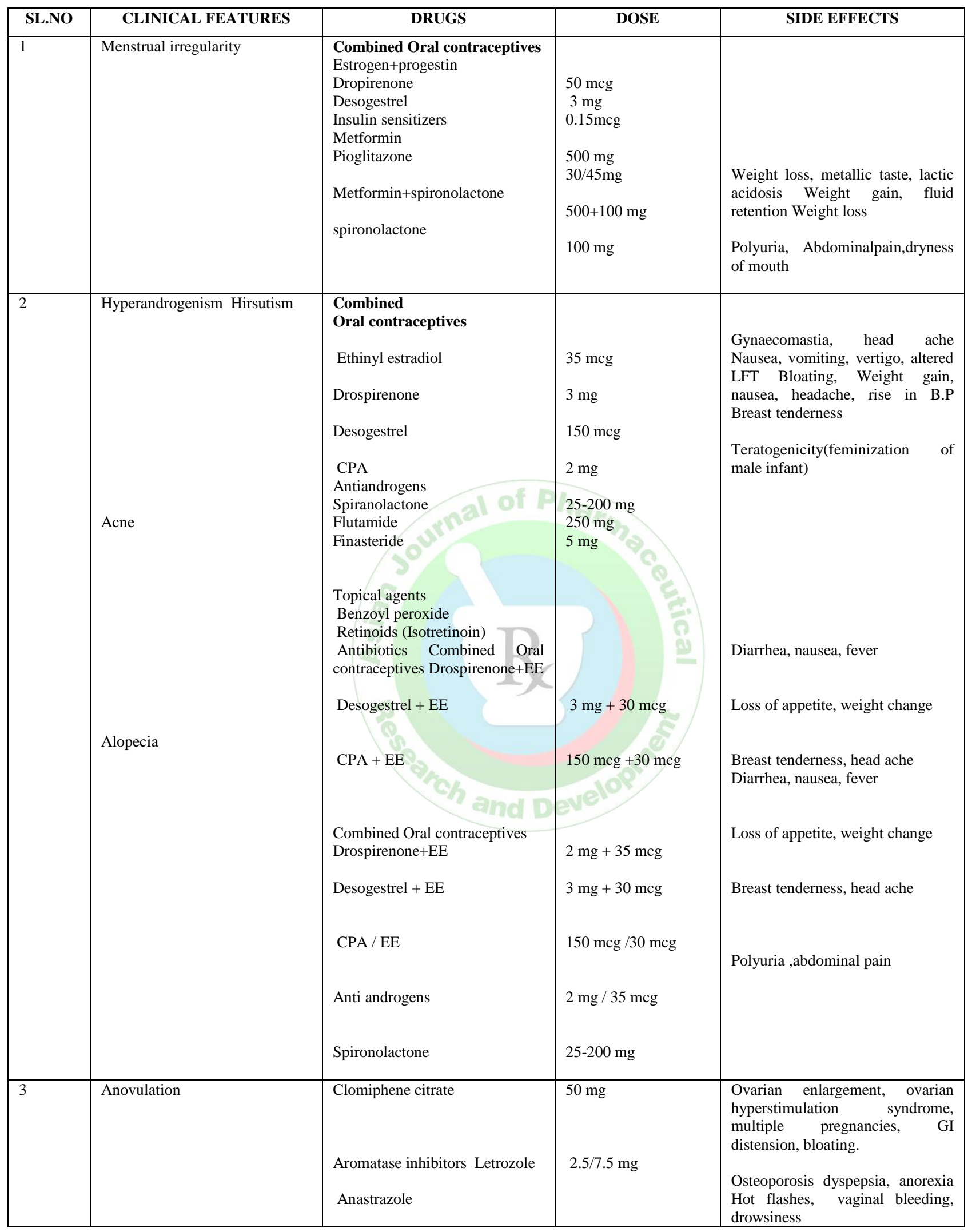

NEWER TREATMENT APPROACHES: According to one study Having long been used as the first-line treatment in PCOS management, combined oral contraceptives
(COCs) offer not just menstrual regulation and endometrial protection, but also a benefit against cutaneous stigmata of hyperandrogenism in women with PCOS.5-7 Mechanisms 
whereby COCs mediate improvements in PCOS-related symptoms include:

A suppression of pituitary luteinizing hormone (LH), thereby reducing the stimulant effect of $\mathrm{LH}$ on androgen production by the ovarian theca cells; An increase in hepatic sex hormone binding globulin (SHBG) directly resulting from the estrogen component in the COC; the net effect is adecline in the free androgen levels and hence an improvement in the clinical features of hyperandrogenism (e.g., acne and hirsutism); and

The antiproliferative effects of the progestin component of the COC formulation, which offers protection against proliferative endometrial pathologies that oligomenorrheic and insulin-resistant women are particularly at risk of. Progesterone-only Approach In oligomenorrheic women, particularly in those with excess body mass, the use of progestins alone (when administered orally or via intramuscular, subcutaneous, or intrauterine delivery routes) can be efficacious in mitigating the risk of endometrial pathologies (such as endometrial hyperplasia and cancer) that are recognized in the setting of chronic annovulation and long-term exposure to unopposed estrogen (endogenous or exogenous). Managing Infertility Impaired functioning of the hypothalamic-pituitary-ovarian (HPO) axis is well recognized to underlie the ovulatory dysfunction of PCOS While oligo-ovulation is an obvious mechanism, other potential contributors to infertility (such as tubal disease and coexisting male factor) must be additionally screened for in the setting of PCOS-related infertility. Selective Estrogen Receptor Modulators Selective estrogen receptor modulators (SERMs) represent the prototype of drugs that are of proven efficacy in achieving ovulation induction. Clomiphene citrate (CC) is conventionally considered as the first-line treatment for the management of the normogonadotropic anovulation seen in PCOS. Tamoxifen, a sister SERM, may be an option in the subgroup of patients who fail to either ovulate or conceive. Aromatase Inhibitors The latest addition to the armamentarium of ovulationinducing agents, aromatase inhibitors (AIs) are increasingly being incorporated into the clinical paradigm for managing ovulatory infertility. Gonadotropins, In patients who are resistant to, or fail to achieve success with, first- and second-line strategies such as SERMs and AIs, ovarian stimulation through exogenous gonadotropins is of proven efficacy in achieving ovulation and reproductive success. Laparoscopic Electrocautery of the Ovaries and/or Laparoscopic Ovarian Drilling For patients with PCOS who have failed to respond to attempts at ovulation induction with first- and second-line strategies such as SERMs and
AIs, bilateral electrocautery of the ovarian surface offers a high likelihood of restoring ovulation ${ }^{35}$

ROLE OF PHARMACIST: Pharmacist can make significant impact on PCOS patients by providing information about disease, lifestyle modifications and medications. Pharmacist plays a vital role in optimizing therapeutic outcomes by counseling patients about appropriate use of medications prescribed, potential adverse effects of medicines and emphasize on importance of medication adherence. Pharmacist should provide additional information about strategies to prevent long term consequences of PCOS. Pharmacist should advice patients to check their blood glucose, lipid profile, BP and weight ${ }^{34}$

\section{CONCLUSION:}

According to recent statistics it was proven that the prevalence of poly cystic ovarian syndrome is increasing rapidly at an alarming rate. More and more approach is needed towards the early diagnosis and treatment of the disease. A pharmacist plays an important role in prevention and management of the disease through counseling the patient basing on the co-morbidities and risk factors. Pharmacist also plays a vital role in prevention of complications associated with polycystic ovarian syndrome.

\section{ABBREVATIONS:}

PCOS-polycystic ovarian syndrome WHO-world health organization CVS-cardiac vascular system LH-leutinising hormone DM-diabetes mellitus

FSH-follicle stimulating hormone

LHR-leutinizing hormone receptor

GnRH-gonadotropin releasing hormone

CRP-c-reactive protein

FPHL-female pattern hair loss

IGF1R-insulin like growth factor receptor 1

AMH-anti-mullerian hormone

HHOA-hypothalamus- hypophysis ovary axis

PAI-plasminogen activator inhibitor

VLDL-very low density lipoprotein

IR-insulin resistance

HDL-C-high density lipoprotein-c

BMI-body mass index

PUFA'S-poly unsaturated fatty acids

AI-aromatase inhibitor

CC-clomiphene citrate

SERMS-selective estrogen receptor modulators

HPO-hypothalamic pituitary ovarian

SHBG-sex hormone binding globulin

COCS-combined oral contraceptives 


\section{REFERRENCES:}

1. Gautam N. Allahbadia, Rubina Merchant. Polycystic ovary syndrome and impact on health. (Middle East Fertility society Journal) 2011; 16:19-37.

2. Uche Anadu Ndefo, PharmD, BCPS; Angie Eaton, PharmD; and Monica RobinsonGreen, BCPS, BCACP. Polycystic Ovary Syndrome A Review of Treatment Options With a Focus on Pharmacological Approaches. (P\&T). 2013; 38(6):336-355

3. V. De Leo, M. C. Musacchio, V. Cappelli, M. G. Massaro, G. Morgante and F. Petraglia. Genetic, hormonal and metabolic aspects of PCOS: an update. (Reproductive Biology and Endocrinology). 2016; 14(38).

4. Choudhary A, Jain S, Chaudhari P Prevalence and symptomology of polycystic ovarian syndrome in Indian women: is there a rising incidence? (International Journal of Reproduction, Contraception, Obstetrics and Gynecology) 2017; 6 (11):4971-4975.

5. Susan M Sirmans Kristen A Pate. Epidemiology, diagnosis, and management of polycystic ovary syndrome. (Dovepress) 2014; 6: 1-13.

6. Wołczyński S, Zgliczyński W. Abnormalities of the menstrual cycle. In: Large Interna - Endocrinology. 2nd edition. Medical Tribune Poland, Warsaw 2012, 561-567.

7. The Rotterdam ESHRE/ASRM-sponsored PCOS consensus workshop group. Revised 2003 consensus on diagnostic criteria and long-term health risks related to polycystic ovary syndrome (PCOS). Hum Reprod. 2004; 19(1):41-47. 10.1093/humrep/deh098.

8. Milewicz A. Reimbursement of metformin for polycystic ovary syndrome. Endokrynol Pol. 2013; 64(5):409-414. 10.5603/EP.2013.0025.

9. Morotti E, Battaglia B, Fabbri R, Paradisi R, Venturoli S, Battaglia C. Cigarette smoking and cardiovascular risk in young women with polycystic ovary syndrome. Int J Fertil Steril. 2014; 7(4):301-312.

10. Davies MJ, Marino JL, Willson KJ, March WA, Moore VM. Intergenerational associations of chronic disease and polycistic ovary syndrome. PLoS One. 2011; 6(10):e25947.

11. Tan S, Scherag A, Janssen OE et al. Large effects on body mass index and insulin resistance women with PCOS (FTO) variants in patients with polycystic ovary syndrome (PCOS). BMC Med Genet. 2010; $11: 12$.

12. Fica S, Albu A, Constantin M, Dobri GA, Davila C. Insulin resistance and fertility in polycystic ovary syndrome. J Med Life. 2008; 1(4):415422 .

13. Traub ML. Assessing and treating insulin resistance in women with polycystic ovarian syndrome. World J Diabetes. 2011; 2(3):33-40.

14. Sathyapalan T, Atkin ST. Mediators of inflammation in polycystic ovary syndrome in relation to adiposity. Mediat Inflamm. 2010; 2010:

15. Kharitonenkov A, Adams AC. Inventing new medicines: The FGF21 story. Mol Metab. 2014; 3:221-229. 10.1016/j.molmet.2013.12. 003.

16. McClung J, Karl P. Iron deficiency and obesity: The contribution of inflammation and diminished iron absorption. Nutr Rev. 2009; 67: 100104.

17. Bentley-Lewis R, Selly E, Dunaif A. Ovarian hypertension: Polycystic ovary syndrome. Endocrinol Metab Clin North Am. 2011; 40:433-449.

18. Archer JS, Chang RJ. Hirsutism and acne in polycystic ovary syndrome. Best Pract Res Clin Obstet Gynaecol 2004; 18:737-54.
19. Azziz R. The evaluation and management of hirsutism. Obstet Gynecol 2003; 101:995-1007.

20. Morin-Papunen LC, Vauhkonen I, Koivunen RM, Ruokonen A, Martikainen HK, Tapanainen JS. Endocrine and metabolic effects of metformin versus ethinyl estradiol-cyproterone acetate in obese women with polycystic ovary syndrome: A randomized study. J Clin Endocrinol Metab 2000; 85:3161-8.

21. Olsen EA. Female pattern hair loss. J Am Acad Dermatol 2001; 45:7080.

22. Park JC, Lim SY, Jang TK, Bae JG, Kim JI, Rhee JH. Endometrial histology and predictable clinical factors for endometrial disease in women with polycystic ovary syndrome. Clin Exp Reprod Med 2011; $38: 42-6$

23. Samer ElHayek1, LynnBitar, LayalH.Hamdar1, FadiG.Mirza and Georges Daoud1. Poly Cystic Ovarian Syndrome: An Updated Overview. (Frontiers in Physiology) 2016; 7(124).

24. Joselyn Rojas, Mervin Chávez, Luis Olivar Milagros Rojas, JesseniaMorillo, JoséMejías, María Calvo, and Valmore Bermúdez1. Polycystic Ovary Syndrome, Insulin Resistance, and Obesity: Navigating the Pathophysiologic Labyrinth. (International Journal of Reproductive Medicine) 2014

25. Dinka Pavicic Baldani, Lana Skrgatic, and Roya Ougouag. Polycystic Ovary Syndrome: Important Underrecognised Cardiometabollic Risk Factor in Reproductive- Age Women. (International Journal of Endocrinology) 2015

26. Susan Sam, MD. Obesity and Polycystic Ovary Syndrome. (NIH Obes Manag) 2007; 3(2): 69-73.

27. Daniilidis A, Dinas K. Long term health consequences of polycystic ovarian syndrome: a review analysis. 2009; 13(2): 90-92.

28. Stefano Palomba, Susanna Santagni, et al. Complications and challenges associated with cPolycysti ovary syndrome: current perspectives. (International Journal of Women's Health) 2015; 7:745-763.

29. Andrea Dunaif. Insulin Resistance and the Polycystic Ovary Syndrome:Mechanism and Implications for Pathogenesis. (Endocrine Reviews) 18(6): 774-800.

30. Crystal C. Douglas, Ph.D., R.D.a,b, Barbara A. Gower, Ph.D.a, Betty E Darnell, M.S., R.D.b,et al. Role of diet in the treatment of polycystic ovary syndrome. (American Society for Reproductive Medicine) 2006; 85(3):679-688

31. H. Farshchi, A. Rane, A. Love \& R. L. Kennedy. Diet and nutrition in polycystic ovary syndrome (PCOS): Pointers for nutritional management. (Journal of Obstetrics and Gynaecology); 27(8):762 773.

32. Artemis aki, 1Karkanoannis Kalogiannidis and Dimitrios Panidis Effects of Lifestyle Modification on Pregnancy Success in Obese and Overweight Women with PCOS. (J Women's Health Care) 2013; 2(2).

33. Cheryce L. Harrison, Catherine B. Lombard, Lisa J. Moran , and Helena J. Teede Exercise therapy in polycystic ovary syndrome: a systematic review. (Human Reproduction Update) 2011; 17(2):171-183.

34. Polycystic Ovary Syndrome. (Saskatchewan Drug Information Services) 2004; 21(1)

35. Sanam Lathief, Lubna Pal, Advances In Treatment Options For Polycystic Ovary Syndrome - Us Endocrinology, 2012;8(1):57-64 